\title{
REGULAR AND SINGULAR PERTURBATIONS OF UPPER SEMICONTINUOUS DIFFERENTIAL INCLUSION
}

\section{TZANKO DONCHEV}

and

VASIL ANGELOV

Department of Mathematics

University of Mining and Geology

1100 SOFIA, BULGARIA

(Received November 13, 1995 and in revised form April 11, 1996)

\begin{abstract}
In the paper we study the continuity properties of the solution set of upper semicontinuous differential inclusions in both regularly and singularly perturbed case. Using a kind of dissipative type of conditions introduced in [1] we obtain lower semicontinuous dependence of the solution sets. Moreover new existence result for lower semicontinuous differential inclusions is proved.
\end{abstract}

KEY WORDS AND PHRASES: One side Lipschitz, Lemma of Plis, Singular perturbations. 1991 AMS SUBJECT CLASSIFICATION CODES: 34A60, 34E15, 49J24, 49K24.

\section{INTRODUCTION}

In the paper we consider the following regularly perturbed multivalued differential equation:

$$
\dot{x}(t) \in F(x(t), \alpha), \quad x(0)=x_{0} ; \quad t \in[0,1]
$$

Where $x \in H$ (Hilbert space), $\alpha \in D$ (metric space), $F$ is a multi from $H \times D$ into $H$ and has closed convex bounded images. Moreover $F(., \alpha)$ is upper semicontinuous, $F(x,$.$) is continuous in$ the sense of graph. Let $H=H_{1} \times H_{2}, H_{2}$ is Hilbert $i=1,2$. The following Cauchy problem:

$$
\left(\begin{array}{c}
\dot{x}(t) \\
\epsilon \dot{y}(t)
\end{array}\right) \in F(x, y), \quad x(0)=x_{0}, \quad y(0)=y_{0}
$$

called singularly perturbed is also considered. For $\epsilon=0$ one has

$$
\left(\begin{array}{c}
\dot{x}(t) \\
0
\end{array}\right) \in F(x, y), \quad x(0)=x_{0}
$$

The last system is called reduced inclusion. The pair of $\mathrm{AC} x($.$) and L_{2}-y($.$) is a solution of (1.3),$ when (1.3) holds for a.e. $t$. Suppose $F$ is one side Lipschitz on $x$ we prove that the solution set $Z(\alpha)$ of (1.1) depends continuously on $\alpha$ in $C(I, H)$. In the literature the continuous properties of $Z($.$) are studied when F(., \alpha)$ is Lipschitz (in that case $F($.$) is continuous). So our results are new$ also in case of finite dimensional spaces. For $F(x,$.$) with convex graph the upper semicontinuous$ properties of the solution set of (1.2) are studied in [2]. The lower semicontinuous properties of the last set are studied in [3] under different type of hypotheses then theese of [2]. The existnce 
of Lipschitz solution of (1.3) is proved in [4]. Using refined version of the lemma of Plis, Veliov shows in [3] that the solution set of (1.2) is LSC at $\epsilon=0^{+}$with respect to $C\left(I, R^{n}\right) \times L_{2}\left(I, R^{m}\right)$ topology. In both papers $F$ is assumed to be Lipschitz. In our paper the Lipschitz continuity requirement of $F$ is dispenced with. The LSC of the solution set for more general systems than (1.1) is investigated in [1] for one side Lipschitz $F$. However $F$ is assumed to be continuous. When $F$ is only USC it is difficult to show the existence of solutions when $F$ does not satisfy additional compactness hypotheses. Such a problem is considered in [5] when $H^{*}$ is uniformly convex Banach space. Here we use the techniques developed there (we generalise theorem 1 of [5]). In section 2 we extend the well known lemma of Plis [6]. In paragraph 3 as a trivial consequence of the refined version of the last lemma we show the continuous dependence of $Z($.$) on \alpha$ for (1.1). We also obtain existence result for lower semicontinuous diffrential inclusions which do not satisfy any compactness conditions. In the last section using similar ideas as in [3] we prove the LSC dependence on $\epsilon$ of the solution set of (1.2) at $\epsilon=0^{+}$. We note that the main results in the paper can be proved also for Banach $H$ with uniformly convex dual $H^{*}$.

\section{PRELIMINARIES.}

In the paper $I:=[0, T]$ (commonly $T=1$ ), $H$ (for system (1.2) $H=H_{1} \times H_{2}$ ) is a Hilbert space with scalar product $\langle.,$.$\rangle , while \sigma(x, A)$ is the support function $\sup _{a \in A}\langle x, a\rangle$. The graph of the multi $F: H \rightarrow P_{f}(H)$ (Nonempty closed convex bounded subsets of $H$ ) is the set Graph $F:=\{(x, y) \in H \times H: y \in F(x)\}$. When this set is closed in $H \times H$ we say that $F$ has a closed graph. We denote by $d(x, A)=\inf \{|x-y|: y \in A\}$. The Hausdorff distance is $D_{H}(A, B):=\max \left\{\sup _{a \in A} d(a, B), \sup _{b \in B} d(b, A)\right\}$. The multi $F$ is called USC (LSC) at $x$, when to $\epsilon>0$ there exists $\delta>0$ such that $F(x)+\epsilon U \supset F(y)) ;(F(x) \subset F(y)+\epsilon U)$ whenever $|x-y| \leq \delta$. Here $U=\{x:|x| \leq 1\}$. The multi $F$ from $I \times E$ into $P_{f}(E)$ is said to be almost upper (lower) semicontinuous (AUSC) if to $\epsilon>0$ there exists $I_{\epsilon}$ with meas $\left(I \backslash I_{\epsilon}\right)>1-\epsilon$ such that $F$ is USC (LSC) on $I_{\mathrm{c}} \times E$. The Lipschitz function $x$ with constant $\leq N$ will be called $N$-Lipschitz. For the system (1.2) we will use the following hypotheses:

A1. $F(.,$.$) is USC, closed convex valued bounded on bounded sets.$

A2. (One side Lipschitz condition) There exist positive constants $L_{1}, L_{2}, L_{3}, \mu$.

If $\left(x_{1}, y_{1}\right),\left(x_{2}, y_{2}\right) \in H_{1} \times H_{2}$ and $f \in F\left(x_{1}, y_{1}\right)$, then there exists $g \in F\left(x_{2}, y_{2}\right)$ such that:

$$
\begin{aligned}
& <x_{1}-x_{2}, f^{*}-g^{*}>\leq L_{1}\left|x_{1}-x_{2}\right|^{2}+L_{2}\left|x_{1}-x_{2}\right|\left|y_{1}-y_{2}\right| \\
& <y_{1}-y_{2}, f^{\prime}-g^{\prime}>\leq L_{3}\left|x_{1}-x_{2}\right|\left|y_{1}-y_{2}\right|-\mu\left|y_{1}-y_{2}\right|^{2} .
\end{aligned}
$$

Here $f^{*}$ and $f^{\prime}$ are the projections of $f$ on $H_{1}$ and $H_{2}$ respectively.

REMARK. Obviously if $F(x, y)=F_{1}(x, y) \times F_{2}(x, y)$ then A2 becomes:

$$
\begin{aligned}
& \sigma\left(x_{1}-x_{2}, F_{1}\left(x_{1}, y_{1}\right)\right)-\sigma\left(x_{1}-x_{2}, F_{1}\left(x_{2}, y_{2}\right)\right) \leq \\
& L_{1}\left|x_{1}-x_{2}\right|^{2}+L_{2}\left|x_{1}-x_{2}\right|\left|y_{1}-y_{2}\right| \\
& \sigma\left(y_{1}-y_{2}, F_{2}\left(x_{1}, y_{1}\right)\right)-\sigma\left(y_{1}-y_{2}, F_{2}\left(x_{2}, y_{2}\right)\right) \leq \\
& L_{3}\left|x_{1}-x_{2}\right|\left|y_{1}-y_{2}\right|-\mu\left|y_{1}-y_{2}\right|^{2} .
\end{aligned}
$$

A2 is a one-side Lipschitz condition combined with a stability-type condition. If the $y$ part of (1.1) has the form

$$
f(y(t))+V(x(t))
$$

then $\mathrm{A} 2$ is equivalent of $f$ is dissipative, i.e.

$$
<y_{1}-y_{2}, f\left(y_{1}\right)-f\left(y_{2}\right)>\leq-\mu\left|y_{1}-y_{2}\right|^{2}
$$


and $V($.$) is Lipschitz. If f(x)=A x$ ( $f$ is linear) and $H$ is finite dimensional then A2 is fulfilled, when the eigenvalues of the matrix $A$ have negative real parts. Various prototypes of A2 are common in the singular perturbation literature.

PROPOSITION 2.1. Let A1, A2 hold. then there exist constants $k_{x}, k_{y} ; M$ such that $\left|x_{\iota}(t)\right| \leq k_{x} ;\left|y_{\epsilon}(t)\right| \leq k_{y}$ and $\left|F\left(x_{\iota}, y_{\epsilon}\right)\right| \leq M, t \in I$ for every $\epsilon>0$ and every AC $\left(x_{c}, y_{\epsilon}\right)$ with $d\left[\left(x_{\iota}, y_{\epsilon}, \dot{x}_{\epsilon}, \epsilon \dot{y}_{\epsilon}\right), G r a p h F\right] \leq 1$

PROOF. Using standard arguments [7], [3] one can show that there exist $r, s$ such that $r \geq\left|x_{\epsilon}\right|^{2}, s \geq\left|y_{\epsilon}\right|^{2}$ and

$$
\begin{array}{ll}
\dot{r}=C_{1} r+C_{2} s+C_{3}, & r(0)=\left|x_{\epsilon}(0)\right|^{2} \\
\epsilon \dot{s}=D_{1} r-\mu s+D_{2}, & s(0)=\left|y_{\epsilon}(0)\right|^{2}
\end{array}
$$

where $C_{1}, C_{2}, C_{3}, D_{1}$ and $D_{2}$ are positive constants. Since $s \leq \mu_{1}\left(D_{1} r+D_{2}\right)$ or $\dot{s}<0$ one has that $r \leq \exp \left(C_{1}+C_{2} D_{1} / \mu\right)\left(C_{3}+r(0)\right)$ and $s \leq \mu_{1}\left(D_{1} r+D_{2}\right)+s(0)$. QED.

REMARK. In view of proposition 2.1 we suppose $|F(x, y)| \leq M$, since we consider only AC functions $(x, y)$, satisfying the conditions of proposition 2.1 .

The following lemma extend the well known lemma of Plis [6]. Using similar arguments as in [5] we relax the continuity and Lipschitz assumptions of [6] and refine the estimation as well.

LEMMA 2.1. Let $d\left[\left(x_{\epsilon}, y_{\epsilon}, \dot{x}_{\epsilon}, \epsilon \dot{y}_{\epsilon}\right) . G r a p h F\right] \leq \delta$ and let $y_{\epsilon}$ be $N$-Lipschitz. Then for every ' $\lambda>0$ there exists a solution $(x, y)$ of (1.2) such that $\left|x(t)-x_{\epsilon}(t)\right|^{2} \leq r_{1}(t)+\lambda ; \quad\left|y(t)-y_{\epsilon}(t)\right|^{2} \leq$ $r_{2}(t)+\lambda$, where $r_{1}$ and $r_{2}$ are the solutions of the system:

$$
\begin{aligned}
& \dot{r}_{1}=4 L_{1} r_{1}+L_{2} r_{2} / L_{1}+C_{1} \delta \quad r_{1}(0)=\left|x_{\epsilon}(0)-x(0)\right| \\
& \dot{r}_{2}=\epsilon^{-1} \mu^{-1}\left\{2 L_{3} r_{1}-\mu^{2} r_{2}+C_{2} \delta\right\} \quad r_{2}(0)=\left|y_{\epsilon}(0)-y(0)\right|
\end{aligned}
$$

where $C_{1}$ and $C_{2}$ are constants (depend on $M$ and $N$, but not on $\delta$ ).

PROOF. Fix $\nu>0$. We claim that there exist $M$ - Lipschitz $u($.$) and M / \epsilon$ - Lipschitz $v($.$) such$ that $d[(u, v, \dot{u}, \epsilon \dot{v}), G r a p h F] \leq \nu$ and moreover the following inequalities hold:

$$
\begin{array}{cl}
\left|u(t)-x_{\epsilon}(t)\right|^{2} \leq m(t) ; \quad\left|v(t)-y_{\epsilon}(t)\right|^{2} \leq n(t), \text { where } \\
\dot{m}(t)=4 L_{1} m+L_{2} n / L_{1}+C_{1}(\delta+\nu), & m(0)=\left|x_{\epsilon}(0)-u(0)\right|^{2} \\
\dot{n}(t)=\epsilon^{-1}\left\{2 L_{3} m-\mu n+C_{2}(\delta+\nu)\right\}, & n(0)=\left|y_{\epsilon}(0)-v(0)\right|^{2} .
\end{array}
$$

Obviously the claim holds for $t=0$. Suppose that it also holds on $[0, \tau]$ with $\tau \geq 0$. If $\tau<1$, then we let by A2 $(f(t), g(t)) \in F(u(\tau), v(\tau))$ to be strongly measurable such that for $\left|x-x_{\ell}\right| \leq \delta,\left|y-y_{\epsilon}\right| \leq \delta$ the following inequalities are valid:

$$
\begin{aligned}
& <x-u(\tau), \dot{x}_{\epsilon}(t)-f(t)>\leq L_{1}\left|u(\tau)-x_{\epsilon}(t)\right|^{2}+L_{2}\left|u(\tau)-x_{\epsilon}(t)\right|\left|v(t)-y_{\epsilon}(t)\right|+C_{1} \delta|x-u(\tau)| . \\
& \epsilon<y-v(\tau), \dot{y}_{\epsilon}(t)-g(t)>\leq L_{3}\left|u(\tau)-x_{\epsilon}(t)\right|\left|v(\tau)-y_{\epsilon}(t)\right|-\mu\left|v(\tau)-y_{\epsilon}(t)\right|^{2}+C_{2} \delta|y-v(\tau)| .
\end{aligned}
$$

The existence of such $f(),. g($.$) follows immediately by A2, when \dot{x}_{\epsilon}(),. \dot{y}_{\epsilon}($.$) are simple functions,$ because $F(u(\tau), v(\tau))$ is fixed set. The general case is a trivial consequence of the fact that every strongly measurable function is an uniform limit of simple functions. Since $|.-$.$| and \langle.,$.$\rangle are$ continuous there exists $\tau^{\prime}>\tau$ such that denoting $u(t)=u(\tau)+\int_{\tau}^{t} f(s) d s ; v(t)=v(\tau)+1 / \epsilon \int_{\tau}^{t} g(s) d s$, one obtains

$$
\begin{aligned}
& <x_{\epsilon}(t)-u(t), \dot{x}_{\epsilon}(t)-\dot{u}(t)>\leq L_{1}\left|u(t)-x_{\epsilon}(t)\right|^{2}+L_{2}\left|u(t)-x_{\epsilon}(t)\right|\left|v(t)-y_{\epsilon}(t)\right| \\
& +C_{1} \delta\left|x_{\epsilon}(t)-u(t)\right|+2 \delta M . \\
& \epsilon<y_{\epsilon}(t)-v(t), \dot{y}_{\epsilon}(t)-\dot{v}(t)>\leq L_{3}\left|u(t)-x_{\epsilon}(t)\right|\left|v(t)-y_{\epsilon}(t)\right|-\mu\left|v(t)-y_{\epsilon}(t)\right|^{2} \\
& +C_{2} \delta\left|y_{\epsilon}(t)-v(t)\right|+2 \delta M .
\end{aligned}
$$


becauce $u($.$) is M$ - Lipschitz and $v($.$) is M / \epsilon$-Lipschitz. Therefore:

$$
\begin{aligned}
& \frac{1}{2} \frac{d}{d t}\left|u(t)-x_{\epsilon}(t)\right|^{2} \leq L_{1}\left|u(t)-x_{\epsilon}(t)\right|^{2}+L_{2}\left|u(t)-x_{\epsilon}(t)\right|\left|v(t)-y_{\epsilon}(t)\right|+C_{1}(\delta+\nu) M\left(1+L_{2}\right) . \\
& \frac{1}{2} \frac{d}{d t}|y(t)-v(t)|^{2} \leq L_{3}\left|u(t)-x_{\epsilon}(t)\right|\left|v(t)-y_{\epsilon}(t)\right|-\mu\left|v(t)-y_{\epsilon}(t)\right|^{2}+C_{2} \delta|y-v(\tau)|+C \delta
\end{aligned}
$$

for a.e. $t \in\left[\tau, \tau^{\prime}\right]$. If moreover $\left|\tau^{\prime}-\tau\right|<\nu$, then $d[(u, v, \dot{u}, \epsilon \dot{v})$. Graph $F] \leq \nu$. Thus the clai $\mathrm{m}$ holds also on $\left[0, \tau^{\prime}\right]$ and hence on $[0,1]$. Consider now the sequences $\left\{\lambda_{t}\right\}_{t=1}^{\infty},\left\{\left(x_{i}, y_{t}\right)\right\}_{t=1}^{\infty}$ sucr that denoting $y_{1}=v ; x_{1}=u$ one has $\left|x_{i+1}(t)-x_{\imath}(t)\right|+\left|y_{i+1}(t)-y_{\imath}(t)\right| \leq \lambda_{\imath} . y_{i}$ and $y_{i+1}$ are $N / \epsilon$-Lipschitz. We prove that such sequences exist:

let $d\left[\left(x_{\imath}, y_{\imath}, \dot{x}_{\imath}, \epsilon \dot{y}_{t}\right), G r a p h F\right] \leq \nu_{\imath}$ for $i=1,2, \ldots$ and $\left|x_{i+1}-x_{i}\right| \leq m_{i},\left|y_{i+1}-y_{\imath}\right| \leq n_{i}$, where

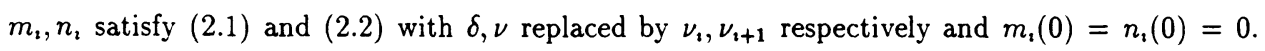
Obviously $\nu_{t}, \nu_{t+1}$ can be chosen such that $\left|m_{t}(t)\right|+\left|n_{t}(t)\right| \leq \lambda_{z}$. (if $\lambda_{t}$ is given). If $\infty_{i=1}^{\infty} \lambda_{t}<\lambda$, then the sequences $\left\{x_{t}\right\}_{i=1}^{\infty}$ and $\left\{y_{t}\right\}_{t=1}^{\infty}$ are Cauchy ones in $C\left(I, H_{1}\right)$ and $C\left(I, H_{2}\right)$ respectively. Obviously their cluster points $x($.$) and y($.$) are solutions satisfying the conclusion of the lemma.$ QED.

In the same fashion one can prove the next variant of lemma 2.1 .

LEMMA 2.2. Let $d\left[\left(x_{\epsilon}, y_{\epsilon}, \dot{x}_{\epsilon}, \epsilon \dot{y}_{\epsilon}\right), G r a p h F\right] \leq \delta$ on $I \times H$ with measI $>1-\delta$ and et $d\left[\left(x_{\epsilon}, y_{\epsilon}, \dot{x}_{\epsilon}, \epsilon \dot{y}_{\epsilon}\right), G r a p h F\right] \leq M$ on $A \times H ; A=I \backslash I$. For every $\lambda>0$ there exists a solution $(x, y)$ of (1.2) such that $\left|x(t)-x_{\epsilon}(t)\right|^{2} \leq r_{1}(t)+\lambda ; \quad\left|y(t)-y_{\epsilon}(t)\right|^{2} \leq r_{2}(t)+\lambda$, where $r_{1}$ and $r_{2}$ are the solutions of the system:

$$
\begin{aligned}
& \dot{r}_{1} \leq 4 L_{1} r_{1}+L_{2} r_{2} / L_{1}+C_{1}(\delta+\alpha(t)) \quad r_{1}(0)=\left|x_{\epsilon}(0)-x(0)\right| \\
& \dot{r}_{2} \leq \epsilon^{-1} \mu^{-1}\left\{2 L_{3} r_{1}-\mu^{2} r_{2}+C_{2}(\delta+\alpha(t))\right\} \quad r_{2}(0)=\left|y_{\epsilon}(0)-y(0)\right|
\end{aligned}
$$

Here $\alpha(t)=M, t \in A$; and $\alpha(t)=0$ otherwise.

The only different step is to prove the existence of $u($.$) and v($.$) such that$

$$
\begin{aligned}
& \left|u(t)-x_{\epsilon}(t)\right|^{2} \leq m(t), \quad\left|v(t)-y_{\epsilon}(t)\right|^{2} \leq n(t), \quad d[(u, v, \dot{u}, \epsilon \dot{v}), \text { GraphF }] \leq \nu \text { and } \\
& \dot{m} \leq 4 L_{1} m+L_{2} n / L_{1}+C_{1}(\delta+\alpha(t)+) \quad m(0)=|u(0)-x(0)| \\
& \dot{n} \leq \epsilon^{-1} \mu^{-1}\left\{2 L_{3} m-\mu^{2} n+C_{2}(\delta+\alpha(t)+)\right\} \quad n(0)=|v(0)-y(0)| .
\end{aligned}
$$

The fashion however is the same and the proof is omitted. QED

Fix $\alpha$ and consider the system (1.1) under the assumptions:

C1. $F($.$) is USC closed convex valued bounded on the bounded sets.$

C2. $\sigma(x-y, F(x))-\sigma(x-y, F(y)) \leq L|x-y|^{2}$. Here $F(x)=F(x, \alpha)$.

COROLLARY 2.1. If $y($.$) is AC function such that d[(y, \dot{y}), G r a p h F] \leq \epsilon$, then there exists a solution $x($.$) of (1.1) such that |x(t)-y(t)|^{2} \leq r(t)+\lambda$, where $r($.$) is the solution of$ $\dot{r}(t)=4 L r+C \epsilon, \quad r(0)=|x(0)-y(0)|$. Here $C$ depends on $M$ (see proposition 2.1), but not on $\epsilon$.

REMARK. When $H=R^{n}$ one can replace $\lambda$ by zero.

\section{REGULARLY PERTURBED CASE.}

Using lemma 2.1 and corollary 2.1 we will prove our main results, which are similar in the regularly and singularly perturbed case.

Let $M$ be metric space and let the parameter $\alpha \in M$. Suppose that $\mathrm{C} 1, \mathrm{C} 2$ hold uniformly on $\alpha$. Let $A \subset H$ be compact. Denote the restriction of $F$ on $A$ by $F_{A}$ and the solution set of (1.1) by $Z(\alpha)$. The following theorem is valid.

THEOREM 3.1. If $\lim _{\alpha \rightarrow B} G r a p h F_{A}(., \alpha)=\operatorname{Graph}_{A}(., \boldsymbol{B})$ for every compact $A \subset H$ in the sense of the Hausdorff distance, then $Z($.$) is LSC. I.e. to every solution x_{\boldsymbol{\beta}}($.$) of \left(1.1_{\boldsymbol{\beta}}\right)$ there exiss 
a net $x_{\alpha}($.$) of solutions of (1.1 \alpha)$ such that $x_{\alpha}($.$) converges uniformly to x_{\boldsymbol{\beta}}($.$) as \alpha \rightarrow \beta$. Moreoer if $\lim _{\boldsymbol{\alpha} \rightarrow \boldsymbol{\beta}} \operatorname{Graph} F(., \alpha)=\operatorname{Graph} F(., \boldsymbol{\beta})$, then $Z($.$) is continuous.$

PROOF. Let $x($.$) be a solution of \left(1.1_{\beta}\right)$. The set

$$
A:=\{z \in H: \exists t \in I: z=x(t)\} \text { is compact. Therefore }
$$

$D_{H}\left(\operatorname{Graph} F_{A}(., \alpha), \operatorname{Graph}_{A}(., \boldsymbol{\beta}) \rightarrow 0\right.$ as $\alpha \rightarrow \boldsymbol{\beta}$. The proof is complete thanks to corollary 2.1 . QED

If $\lim _{\alpha \rightarrow \beta} D_{H}^{+}\left(\operatorname{Graph}_{A}(., \alpha), \operatorname{Graph}_{A}(., B)\right)=0$, where $D^{+}(C, D)=\sup _{c \in C} \inf _{d \in D}|c-d|$, then $Z($.$) is LSC.$

Consider now the following nonautonomous problem.

$$
\dot{x}(t) \in F(t, x), \quad x(0)=x_{0}
$$

H1. For every $x \in H, F(., x)$ admits a strongly measurable selector, $F(t,$.$) has a closed graph,$ $F$ is convex compact valued and $|F(t, x)| \leq K(1+|x|)$.

H2. $\sigma(x-y, F(t, x))-\sigma(x-y, F(t, y)) \leq L|x-y|^{2}$, recall that $H$ is a Hilbert space.

Consider also the discretized version of (3.1).

$$
\dot{x}(t) \in F\left(t, x\left(\tau_{\imath}\right)\right), \quad x(0)=x_{0}, \quad t \in\left[\tau_{i}, \tau_{i+1}\right)
$$

Here $\tau_{i}=i h, h=1 / k$. Denote by $\mathrm{R}(1)$ and $\mathrm{R}(2)$ the solution set of (3.1) and (3.2).

THEOREM 3.2. Under $\mathrm{H} 1-\mathrm{H} 2$ the differential inclusion (3.1) admits nonempty compact solution set. Moreover there exists a constant $C$ with $D_{H}(R(1), R(2)) \leq C h^{1 / 2}$.

PROOF.. First note that there exist $N \geq|F(t, x)|$ and $M \geq|x|$ when

$$
\dot{x} \in F(t, x+U)+U, \quad x(0)=x_{0}
$$

Let $x($.$) be a solution of (3.1). We construct y($.$) on \left[\tau_{i}, \tau_{i+1}\right)$ as follows $\dot{y}(t) \in F\left(t, y\left(\tau_{i}\right)\right)$ is such that

$$
\begin{aligned}
& <x(t)-y\left(\tau_{\imath}\right), \dot{x}(t)-\dot{y}(t)>\leq \quad L\left|x(t)-y\left(\tau_{\imath}\right)\right|^{2} \text {. Therefore } \\
& <x(t)-y(t), \dot{x}(t)-\dot{y}(t)>\leq \quad<x(t)-y\left(\tau_{\imath}\right), x(t)-y(t)>+ \\
& <y\left(\tau_{\imath}\right)-y(t), \dot{x}(t)-\dot{y}(t)>\leq \quad L\left|x(t)-y\left(\tau_{\imath}\right)\right|^{2}+\left(t-\tau_{\imath}\right) N|\dot{x}(t)-\dot{y}(t)| \\
& \leq L|x(t)-y(t)|^{2}+2 M N L h .
\end{aligned}
$$

If $m=|x-y|^{2}$ then $m(t) \leq \exp (2 L t) 4 M N L h$, i.e. $|x(t)-y(t)| \leq 2 \exp (L t)(M N L)^{1 / 2} h^{1 / 2}$. That is $C=2 \exp (L)(M N L)^{1 / 2}$. Let now $y($.$) be a solution of (3.2)$. Consider another partition of $[0,1]$ on subintervals $\left[\tau_{\imath}^{x}, \tau_{\imath+1}^{x}\right), \tau_{j}^{x}=j h_{x}$. Choose

$$
\dot{x}(t) \in F\left(t, x\left(\tau_{\jmath}^{x}\right)\right) \text { with }\left\langle x\left(\tau_{\jmath}^{x}\right)-y\left(\tau_{\imath}\right), \dot{x}(t)-\dot{y}(t)>\leq L\left|x\left(\tau_{\jmath}^{x}\right)-y\left(\tau_{\imath}\right)\right|^{2}\right.
$$

Analogously following inequality holds

$$
<x(t)-y(t), \dot{x}(t)-\dot{y}(t)>\leq L|x(t)-y(t)|^{2}+2 M N L\left(h+h_{x}\right)
$$

Using the construction in the proof of lemma 2.1 one can show that for every such $y($.$) and every$ so $\lambda$ there exists a solution $z($.$) of (3.1)$ such that $|z(t)-y(t)| \leq C h^{1 / 2}+\lambda$. Here $C$ is determined above. Since $F(.,$.$) is compact valued one has that the solution set \mathrm{R}(2)$ of $(3.2)$ is $C(I, H)$ compact and hence the solution set of (3.1) is compact. Thus $\lambda$ can be replaced by 0 . QED 
Obviously using the same fashion and more careful estimations one can prove the variant of theorem 3.2 for Banach $H$ with uniformly convex $H^{*}$.

THEOREM 3.3. Under $\mathrm{H} 1$ and $\sigma(\jmath(x-y), F(t, x))-\sigma(\jmath(x-y), F(t, y)) \leq L|x-y|^{2}$, where $j(x):=\left\{e \in H^{*}:\langle e, x\rangle=|x|^{2}\right.$ and $\left.|e|=|x|\right\}$, the differential inclusion (3.1) admits nonempty compact solution set such that $\lim _{h \rightarrow 0^{+}} D_{H}(R(1), R(2))=0$.

Using this result one can obtain interesting existence result for LSC differential inclusions.

Corollary 3.1. Let $G$ be closed valued almost LSC multi satisfying the inequality of theorem 3.3. Denote $F(t, x):=\bigcap_{\epsilon>0} c l c o\{u: u \in G(t, y):|y-x|<\epsilon\}$. If $F$ satisfies $H 1$ then the following differential inclusion admits a solution

$$
\dot{x}(t) \in G(t, x), \quad x(0)=x_{0}
$$

PROOF. Let $N$ be as in the proof of theorem 3.2. From theorem 2 of [8] we know that there exists a $\Gamma^{N+1}$ continuous selection $g(t, x) \in G(t, x)$. Recall that $f(.,$.$) is called \Gamma^{N+1}$ continuous at $(t, x)$ when $f\left(t_{\imath}, x_{\imath}\right) \rightarrow f(t, x)$ whenever $\left|x_{z}-x\right| \leq(N+1)\left(t_{\imath}-t\right)$ and $t_{\imath} \searrow t$. An obvios modification of the proof of theorem 6.1 of [9] shows the existence of solution of $\dot{x}=g(t, x)$. QED.

REMARK. The question of the approximation of the solution set of (1.1) is studied in [10] for general nonauthonomous systems. We note only that to the author's knowledge all the existence refults in the litherature use compactness conditions on $G$ or the nonemptiness of the interior of clcoG(.,.). (see e.g. $§ 9,10$ of [9])

\section{SINGULARLY PERTURBED CASE.}

In this section we consider the differential inclusion (1.2). The next theorem shows the LSC dependence of $Z(\epsilon)$ at $\epsilon=0^{+}$with respect to $C \times L_{2}$ topology.

THEOREM 4.1. Suppose A1, A2 hold. Let $(x, y)$ be solution of $(1.3)$ and let $y($.$) be$ continuous. If $\tau \in(0,1)$ and if $\delta$ is fixed then there exists $\epsilon(\delta)$ such that to every $\epsilon<\epsilon(\delta)$ we have $\left|x(t)-x_{\epsilon}(t)\right| \leq \delta$ and $\left|y(t)-y_{\epsilon}(t)\right|_{L_{2}} \leq \delta$ for some solution $\left(x_{\epsilon}, y_{\epsilon}\right)$ of $(1.2)$.

Proof. Fix $\lambda>0$ and $\epsilon>0$. Let $z($.) be $N$-Lipschitz function such that $|z(t)-y(t)| \leq \lambda$. Therefore $d[(x, z, \dot{x}, \epsilon \dot{z}), G r a p h F] \leq \lambda+N \epsilon$, since $d[(x, y, \dot{x}, 0), G r a p h F]=0$. From lemma 2.2 there exists a solution $\left(x_{\epsilon}, y_{\epsilon}\right)$ of (1.2) such that $\left|x(t)-x_{\epsilon}(t)\right| \leq r(t),\left|z(t)-y_{\epsilon}(t)\right| \leq s(t)$, where $s^{2}$ and $r^{2}$ are the maximal solutions of the system:

$$
\begin{aligned}
& \left(r^{2}\right)^{\prime}=2 L_{1} r^{2}+2 L_{2} r s+2 M \lambda \quad r(0)=0 \\
& \left(s^{2}\right)^{\prime}=2 L_{3} r s / \epsilon-2 \mu s^{2} / \epsilon+2(M / \epsilon+N) \lambda \quad s(0)=\left|z(0)-y_{0}\right|
\end{aligned}
$$

Let $m \geq r^{2}$ and $n \geq s^{2}$ be such that

$$
\begin{array}{cl}
\dot{m}=3 L_{1} m+L_{2} n / L_{1}+M \lambda & m(0)=0 \\
\dot{n}=2 L_{3} m /(\mu \epsilon)-\mu n / \epsilon+2(M / \epsilon+N) \lambda, & n(0)=\left|z(0)-y_{0}\right|=n_{0}
\end{array}
$$

Then $\dot{m}(t) \geq 0$ for a.e. $t \in I$. Using the Cauchy formulae and integrating by parts one obtains from (4.2) $n(t) \leq \exp (-\mu t / \epsilon) n_{0}+2 \lambda(N \epsilon+M) / \mu+2 L_{3} m / \mu$. From (4.2) one obtains $\dot{m}(t) \leq$ $\left(3 L_{1}+2 L_{3} / \mu\right) m+2 L_{1} \lambda(N \epsilon+M) /\left(L_{2} \mu\right)+\exp (-\mu t / \epsilon) n_{0}$. Denote $c_{1}=3 L_{1}+2 L_{3} / \mu$ and $c_{2}=$ $L_{1}(M+N \epsilon) /\left(L_{2} \mu\right)$. From the Cauchy formulae follows

$$
\begin{aligned}
& m_{1}(t) \leq \exp \left(c_{1} t\right)\left[n_{0} \cdot \int_{0}^{t} \exp \left(\left(-\mu / \epsilon-c_{1}\right) \tau\right) d \tau+c_{2} \lambda . \int_{0}^{t} \exp \left(-c_{1} \tau\right) d \tau\right] \\
& \leq \exp \left(c_{1} t\right)\left[c_{2} \lambda+n_{0} \epsilon / \mu\right]
\end{aligned}
$$


Denote $c_{3}=2\left(M+N \epsilon+2 L_{3} / \mu\right) / \mu ; c_{4}=\exp \left(c_{1} t\right) / \mu$ hence $n(t) \leq \exp (-\mu \tau / \epsilon)+c_{3} \lambda+c_{4} \epsilon$. Thus $n(t) \leq n_{0} \epsilon /(\mu \tau)+c_{3} \lambda+c_{4} \epsilon$ on $[\tau, 1]$ since $\exp (-\mu \tau / \epsilon) \leq \epsilon /(\mu \tau)$. Since $c_{1}, c_{2}, c_{3}$ and $c_{4}$ do not depend on $\delta$ one can find $\lambda$ and $\epsilon$ such that $n(t) \leq \delta$ and $m(t) \leq \delta$. QED.

COROLLARY 4.1. Under A1, A2 the solution set $Z(\epsilon)$ depends lower semicontinuously on $\epsilon$ at $\epsilon=0^{+}$.

PROOF. Let $(x, y)$ be a solution of (1.3). Fix $\delta$ and $\varphi$. Since $y($.$) is bounded one has that$ there exists $K>0$ and $K$ - Lipschitz $z\left(\right.$.) such that $|z(t)-y(t)| \leq \varphi$ on $I_{\boldsymbol{r}}$ and $|z(t)-y(t)| \leq M$ on $A$. Here $I_{\delta}$ and $A$ are as in lemma 2.2. Thus $d[(x, z, \dot{x}, \epsilon \dot{z}), G r a p h F] \leq \delta$ on $I_{\delta} \times H$ with mas $I_{\epsilon}>1-\delta$ and $d[(x, z, \dot{x}, \epsilon \dot{z}), G r a p h F] \leq M$ on $A \times H$ for small $\epsilon$. From lemma 2.2 there exists a solution $(u, v)$ of $(1.2)$ with $|x(t)-u(t)|^{2} \leq r_{1}(t)+\lambda ; \quad|z(t)-v(t)|^{2} \leq r_{2}(t)+\lambda$, where $r_{1}$ and $r_{2}$ are the solutions of the system:

$$
\begin{aligned}
& \dot{r}_{1} \leq 4 L_{1} r_{1}+L_{2} r_{2} / L_{1}+C_{1}(\delta+\alpha(t)) \quad r_{1}(0)=\left|x_{\epsilon}(0)-x(0)\right| \\
& \dot{r}_{2} \leq \epsilon^{-1} \mu^{-1}\left\{2 L_{3} r_{1}-\mu^{2} r_{2}+C_{2}(\delta+\alpha(t))+K \epsilon\right\} \quad r_{2}(0)=\left|y_{\epsilon}(0)-y(0)\right|
\end{aligned}
$$

One has only to prove that $\left(r_{1}, r_{2}\right)$ converges to zero in $C \times L_{2}$ as $\epsilon \rightarrow 0$, which is standard and is omitted. QED.

EXAMPLE 4.1. Consider the system

$$
\begin{aligned}
& \dot{x}(t) \in x^{1 / 3}+|y|+[0,1] \quad x(0)=0 . \\
& \epsilon \dot{y}(t) \in-y+[0,1] \quad y(0)=1 .
\end{aligned}
$$

Obviously the solution set of this system is not LSC at $\epsilon=0$, because the first inclusion is not Lipschitz. Consider however

$$
\begin{aligned}
& \dot{x}(t) \in-x^{1 / 3}+|y|+[0,1] \quad x(0)=0 . \\
& \epsilon \dot{y}(t) \in-y+[0,1] \quad y(0)=1 .
\end{aligned}
$$

The solution set of last system is LSC since theorem 4.1 holds, however the right-hand side is not Lipschitz. This is true also for the first inclusion (without $y($.$) and without singular perturbation).$ In that case theorem 3.2 is valid.

As we have seen the LSC dependence on parameters in regulary and singulary perturbed cases can be investigated under the same approach. The USC dependence however can not. We give an example for system which is not USC at $\epsilon=0^{+}$.

EXAMPLE 4.2. Consider the system

$$
\begin{aligned}
& \epsilon \dot{x}(t)=-x+w(t) \quad x(0)=0, \text { here } w \in[-1,1] . \\
& \epsilon \dot{y}(t)=-2 y+w(t) \quad y(0)=0
\end{aligned}
$$

For $\epsilon=0$ the solution set of this system is $R(t)=(w(t), w(t) / 2)$ where $w($.$) is arbitrary measurable$ $w(t) \in[-1,1]$. Let $w_{n}(t)=1, t \in[(2 k) /(2 n),(2 k+1) /(2 n)) ; \quad w_{n}(t)=-1$ otherwise. Consider the sequence $\epsilon_{n}=1 /(2 n)$. Let $\left(x_{n}, y_{n}\right)$ be the solution of the system for $w=w_{n}$. It is easy to show that $\lim _{n} 0^{+} \int_{0}^{1}\left|x_{n}(t)-2 y_{n}(t)\right| d t \geq(e-1)^{4} /\left(e^{4}-1\right)$. Thus the solution set of this system does not depend USC on $\epsilon$ at $0^{+}$in $L_{1}-$ strong topology (of course $x_{n}-2 y_{n} \rightarrow 0$ in $L_{2}$-weak). This example is studied in [7].

\section{CONCLUSION REMARK.}

We note that using the properties of the duality map $j($.$) (see theorem 3.3$ for definition and [9] for the properties) one can prove similar results as theorem 3.1 and theorem 4.1 in case of 
uniformly convex Banach space $H^{*}$. Using technique as in the proof of theorem 3.2 and by more carefull estimations one can obtain similar results also in case of nonautonomous system.

ACKNOWLEDGMENT. The research is partialy supported by National Fund for Scientific Research at the Bulgarian Ministry of Science and Education under contract MM 442/94.

\section{REFERENCES}

[1] DONCHEV, T., "Functional Differential Inclusions with Monotone Right Hand Side," Nonlinear Analysis TMA 16 (1991), 533-542.

[2] DONTCHEV, A. and SLAVOV, I., "Upper Semicontinuity of Solutions of Singularly Perturbed Differential Inclusions," in H.-J. Sebastian and K. Tammer (eds.), System Modeling and Optimization, Lect. Notes in Control and Inf. Sc., 14 Springer 1991, 273-280.

[3] VELIOV, V., "Differential Inclusions with Stable Subinclusions," Nonlınear Analysıs TMA. 23 (1994). 910-919.

[4] DONTCHEV, A., DONCHEV, T. and SLAVOV, I., "On the Upper Semicontinuity of the Set of Solutions of Differential Inclusions with a Small Parameter in the Derivative," Nonlinear Analysis TMA 24 (1995) 1.

[5] DONCHEV, T. and IVANOV R., "On the Existence of Solutions of Differential Inclusions in Uniformly Convex Banach Spaces," Math. Balkanica 6 (1992), 13-24.

[6] PLIS, A., "On Trajectories of Orientor Fields," Bull. Acad. Pol. Sci. 1 (1965), 571-573.

[7] DONCHEV, T. and SLAVOV, I., "Singularly Perturbed Functional Differential Inclusions," Set Valued Analysis 3 (1995), 113-128.

[8] BRESSAN, A. and COLOMBO, G., "Selections and Representations of Multifunctions in Paracompact Spaces," Studıa Math. 102 (1992), 209-216.

[9] Deimling, K., "Multivalued Differential Equations," De Gruyter Berlin 1992.

[10] DONTCHEV, A. and FARKHI, E., "Error Estimates for Discretized Differential Inclusions," Computing 41 (1989) 349-358. 


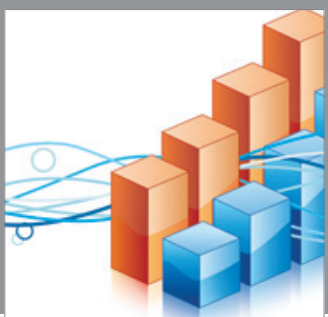

Advances in

Operations Research

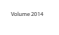

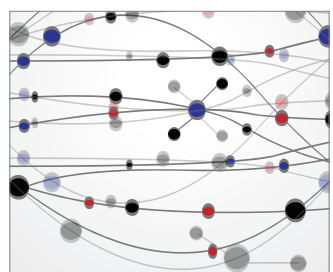

\section{The Scientific} World Journal
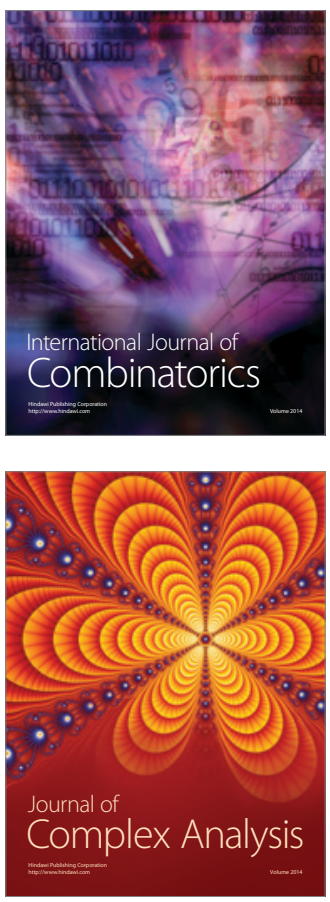

International Journal of

Mathematics and

Mathematical

Sciences
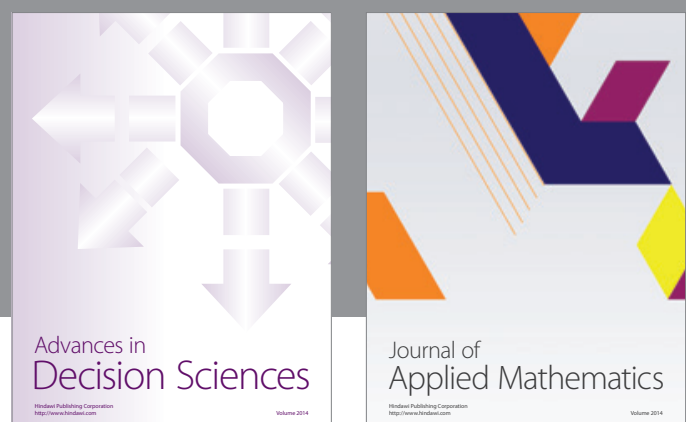

Journal of

Applied Mathematics
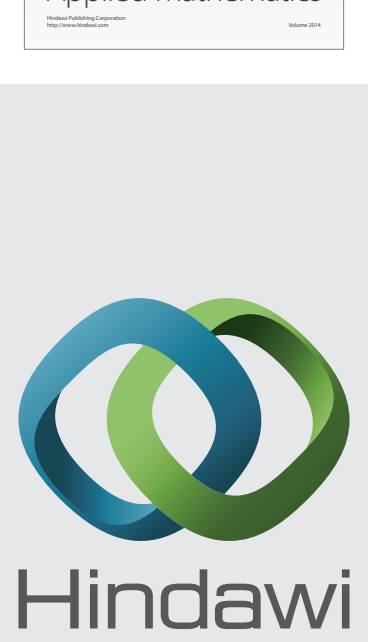

Submit your manuscripts at http://www.hindawi.com
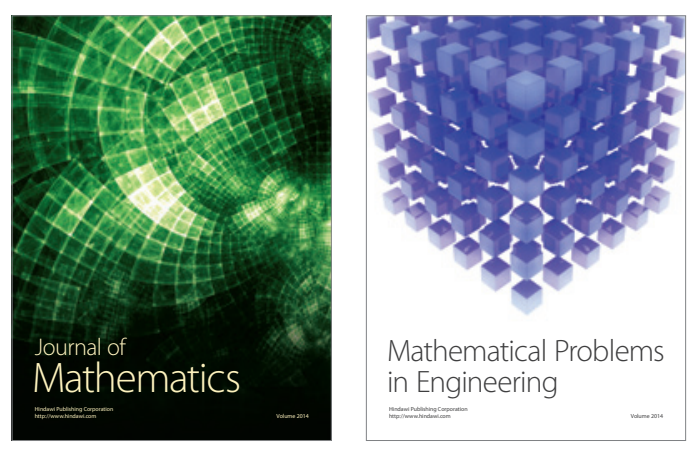

Mathematical Problems in Engineering
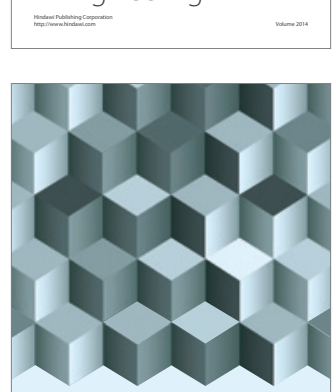

Journal of

Function Spaces
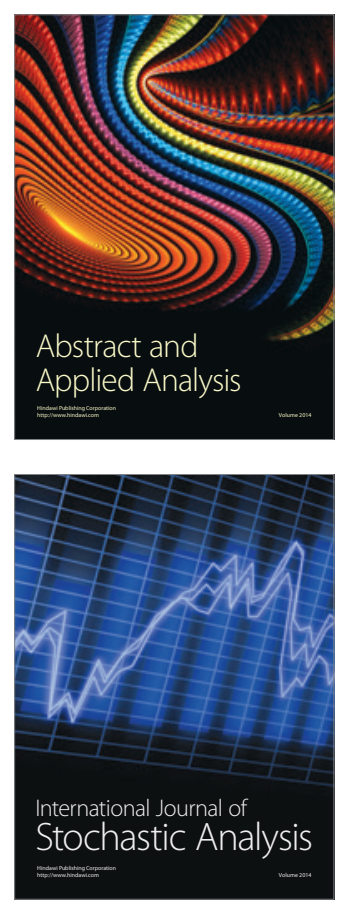

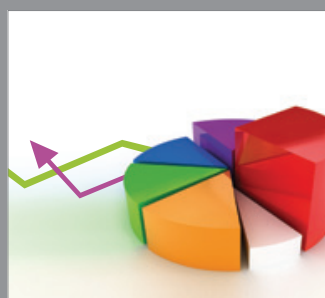

ournal of

Probability and Statistics

Promensencen
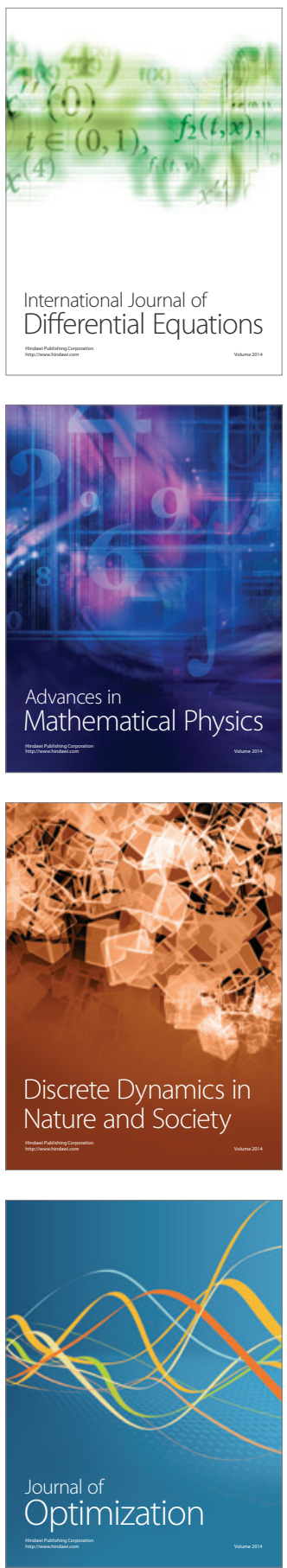